\title{
High-throughput cassette assay for drug stability measurement in plasma using direct HPLC-MS/MS
}

\author{
Gangfeng Wang, Yunsheng Hsieh *, K.-C. Cheng, Kwokei Ng and Walter A. Korfmacher \\ Drug Metabolism and Pharmacokinetics Department, Schering Plough Research Institute, \\ 2015 Galloping Hill Road, K-15-3700, Kenilworth, NJ 07033, USA
}

\begin{abstract}
A high-throughput semi-automated procedure for simultaneously stability evaluation of multiple compounds in plasma using direct single column high-performance liquid chromatography (HPLC) combined with tandem mass spectrometry (MS/MS) was developed to eliminate the laborious procedures that are traditionally used for stability studies. Untreated human, monkey, mouse and rat plasma samples containing ten drug components were directly injected into a mixed-functional column that provided both protein removal and chromatographic functionality. Ten test compounds were simultaneously assayed using a tandem mass spectrometer in the positive ion mode using multiple reaction monitoring (MRM). Plasma samples containing ten test compounds were placed in a thermostatic autosampler and then sequentially monitored in one analytical procedure. The time between each injection was set about 7 minutes. The peak responses of the test compounds in individual plasma samples were repeatedly determined every 28 minutes. Drug stability in plasma was indicated by the change of the mass chromatographic peak areas for the test compounds and was observed to be a function of animal species, incubation time and incubation temperature. The potential for matrix ionization suppression on the direct single column HPLC-MS/MS system was also investigated using the post-column infusion technique. The proposed cassette assay procedure provides an analytical throughput ten times greater than the single component approach for the evaluation of drug stability in plasma without compromising data quality.
\end{abstract}

\section{Introduction}

The stability of new chemical entities (NCE's) in plasma is a concern in the drug discovery area [1-3]. Plasma stability results could be useful in drug discovery programs for the identification of lead molecules and the subsequent optimization of chemical structure, leading the way to clinical candidates with desirable pharmacokinetic (PK) properties. Conventionally, drug stability in plasma has been measured using tedious sample preparation procedures including sequential plasma extraction at each incubation time point [4]. This labor-intensive approach is not suitable for the evaluation of a large number of bioactive compounds, which are generated by medicinal chemists in the modern pharmaceutical industry. Recently, we proposed a semi-automated procedure using direct single column HPLC/MS/MS with the autosampler as the incubator to perform all the functions required for plasma stability determination for a single compound [5]. In the previously described example, we were able to automatically and sequentially monitor the response changes of the test compound in rat, mouse, monkey and human plasma samples as a function of incubation time. However, the tremendous influx of many potent new chemical entities (NCE's) produced weekly in today's pharmaceutical industry demands an even higher throughput procedure for plasma stability experiments.

\footnotetext{
*Corresponding author. Tel: +1 908740 585; E-mail: yunsheng.hsieh@spcorp.com.
} 
In this work, we investigated a cassette assay procedure combined with the direct HPLC-MS/MS approach for an even higher-throughput screen-type assay to simultaneously measure drug stability of multiple drug candidates in several plasma types. The stability results of ten test compounds in rat, mouse, monkey and human plasma obtained by the conventional manual procedures using protein-precipitation method and the proposed semi-automated method using cassette assay procedure are found to be in a good agreement. In addition, although many direct plasma injection methods using HPLC-MS/MS systems were explored for the quantitative determination of pharmaceutical components [6-12], there has been little investigation on the matrix ionization suppression effect in these reports. In this presentation, we employed the post-column infusion technique $[13,14]$ to study the matrix ionization suppression effects on the proposed single column direct plasma injection method.

\section{Experimental}

\subsection{Reagents and chemicals}

The test compounds \# 1 through \# 9 with molecular weights ranging from approximately 300 to 800 are new chemical entities made by Schering Plough Research Institute chemists. The chemical structure of compound \# 1 was published elsewhere [15]. Clozapine was purchased from Sigma (St. Louise, MO, USA). A stock solution containing all ten test compounds $(1 \mathrm{mg} / \mathrm{ml})$ was prepared in methanol. Acetonitrile and triflouroacetic acid (TFA) (HPLC grade) was purchased from Fisher Scientific (Pittsburgh, PA, USA). Acetic acid and ammonium acetate was purchased from Aldrich Chemical Company, Inc. (Milwaukee, WI, USA). Deionized water was generated from a Milli-Q water purifying system purchased from Millipore Corporation (Bedford, MA, USA) and house high-purity nitrogen (99.999\%) was used. Drug-free human, monkey, mouse and rat plasma samples were purchased from Bioreclamation Inc. (Hicksville, NY, USA). Mobile phases A and B are $4 \mathrm{mM}$ ammonium acetate and $0.01 \%$ TFA in water-acetonitrile $(90: 10)$ and $4 \mathrm{mM}$ ammonium acetate in water-acetonitrile $(10: 90)$, respectively.

\subsection{Equipment}

LC-MS/MS analysis was performed using a PE Sciex (Concord, Ontario, Canada) Model API 3000 triple quadrupole mass spectrometer equipped with an atmospheric pressure chemical ionization (APCI) interface (heated nebulizer). The HPLC system consisted of a Leap autosampler (also used as the incubator) with a sample tray set at $21^{\circ} \mathrm{C}$ (LEAP Technologies, Carrboro, NC), Shimadzu on-line degasser, LC-10AD VP pump and LC-10A VP controller (Columbia, MD, USA). For the traditional procedure using the protein precipitation technique, a Synergi C18 column $(2.0 \times 30 \mathrm{~mm}, 4 \mu)$ from Phenomenex Inc (Torrance, CA, USA) was used as the analytical column [13,14]. For the single column direct plasma injection method, a Capcell MF C8 column $(4.6 \times 50 \mathrm{~mm})$ from Phenomenex Inc (Torrance, CA, USA) was used as the pretreatment and analytical column.

\subsection{Stability plasma preparation}

To investigate the stability of the test compounds in plasma, samples were made by spiking either ten compounds or individual test compounds into the blank plasma from the stock solution to yield a total concentration of $10 \mu \mathrm{M}(1 \mu \mathrm{M}$ for each compound). For the semi-automated determination, the spiked 
plasma samples in a 96-well plate were directly and sequentially injected into the single column HPLCMS/MS system, as reported elsewhere [12]. For the traditional method, the spiked plasma samples were first incubated at the desired temperature, then, every $30 \mathrm{~min}$ a $50-\mu \mathrm{l}$ plasma aliquot from the incubation mixture was placed into a test tube and immediately mixed with $150 \mu \mathrm{l}$ acetonitrile solution for protein precipitation. After centrifugation, the supernatant was then transferred into a 96-well plate for HPLCMS/MS analysis.

\subsection{Chromatographic conditions}

The instrumental configurations for the single column direct plasma injection method had been reported previously [10]. The LC flow rate was constant at $1 \mathrm{ml} / \mathrm{min}$. A $10-\mu 1$ portion of the plasma sample was injected by the autosampler directly onto the CAPCELL MF C8 column with mobile phase A and the divert valve (post-column) switched to waste to remove the macromolecules from the plasma matrix. Macromolecules such as protein passed quickly through the column due to the restricted access to the surface of the packing while the column retained the drug molecules on the bonded hydrophobic phase. After 2 min the column effluent was diverted from waste to the mass spectrometer and the HPLC pump was switched from mobile phase A to mobile phase B over 2.5 min to simultaneously determine the responses of all analytes in plasma in one HPLC run. The retention times for all compounds of interest were less than $4 \mathrm{~min}$. The instrumental setup for the post-column infusion technique to explore the matrix ionization suppression effect of the test compounds in plasma was described elsewhere [13].

\subsection{Mass spectrometric conditions}

The mass spectrometer was operated in the positive ion mode. The heated pneumatic nebulizer probe conditions were as follows: $450^{\circ} \mathrm{C}$ temperature setting, $80 \mathrm{psi}$ nebulizing gas pressure, $1.01 \mathrm{~min}^{-1}$ auxiliary gas flow, $0.91 \mathrm{~min}^{-1}$ curtain gas flow-rate. The MS/MS reactions selected to monitor clozapine and the compounds \# 1 through \# 9 were the transition from $m / z 327,639,624,560,654,477,478,514$, 781 and 634 , to a product ion at $m / z 270,471,447,308,294,358,447,392,682$ and 308, respectively. The product ion spectra of all analetes are shown in Fig. 1. The protonated molecules were fragmented by collision-activated dissociation (CAD) with nitrogen as the collision gas at a pressure of instrument setting 5. The collision-offset voltage was set at $40 \mathrm{~V}$.

\section{Results and discussion}

The advancement of genomics techniques and high speed chemical synthesis results in large volume of routine samples created from pharmacokinetic (PK) and drug metabolism (DM) research in drug discovery. Cassette analysis, simultaneously combining many test compounds into one sample, is an attractive method for reducing the amount of routine samples and increasing the throughput of various in vivo or in vitro DM/PK studies [16-19]. HPLC combined with atomospheric pressure ionization (API) tandem mass spectrometry (MS/MS) is a powerful tool for rapid quantitative method development, metabolite identification and multi-drug analysis enabling the technology for cassette assay [20]. However, sample preparation such as protein precipitation, solvent extraction or solid phase extraction techniques [20] is still the most common procedure prior to HPLC-MS/MS analysis to prevent both HPLC column 
Clozapine

Compound \#

Compound \# 2

Compound \# 3

Compound \# 4

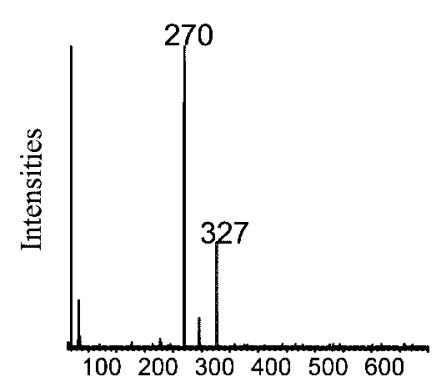

$\mathrm{m} / \mathrm{z}$, amu

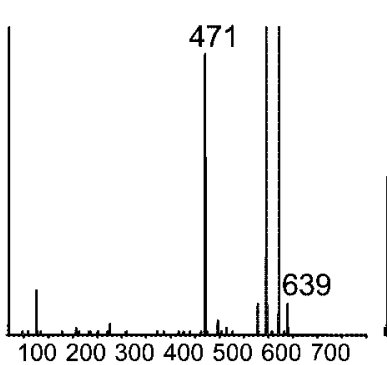

$\mathrm{m} / \mathrm{z}$, amu

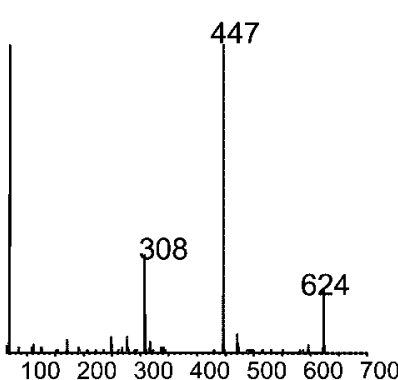

$\mathrm{m} / \mathrm{z}$, amu

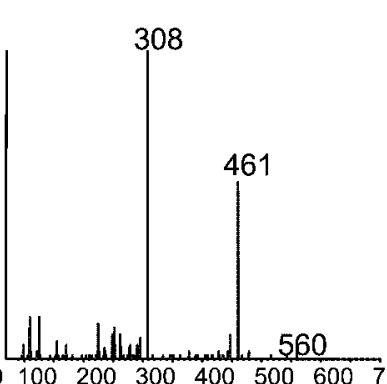

$\mathrm{m} / \mathrm{z}, \mathrm{amu}$

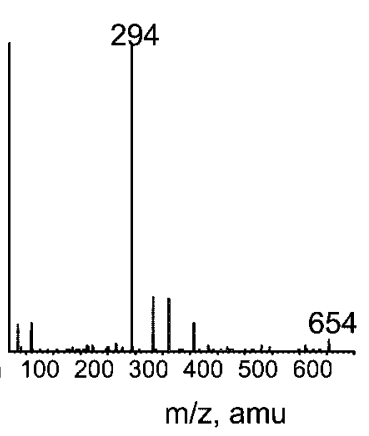

$\mathrm{m} / \mathrm{z}, \mathrm{amu}$

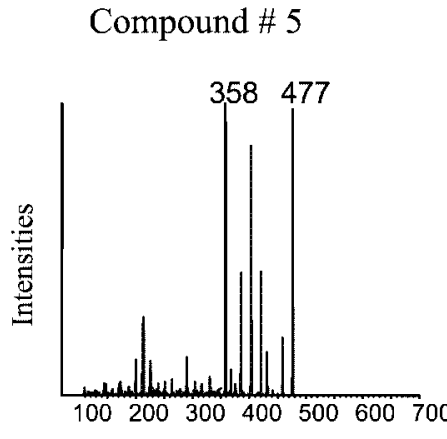

Compound \# 6

Compound \# 7

Compound \# 8

Compound \# 9

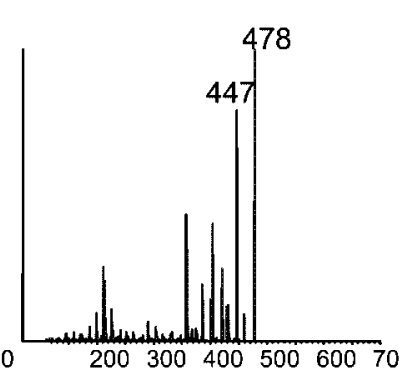

$\mathrm{m} / \mathrm{z}$, amu

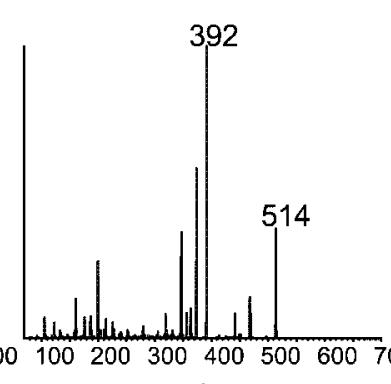

$\mathrm{m} / \mathrm{z}$, amu

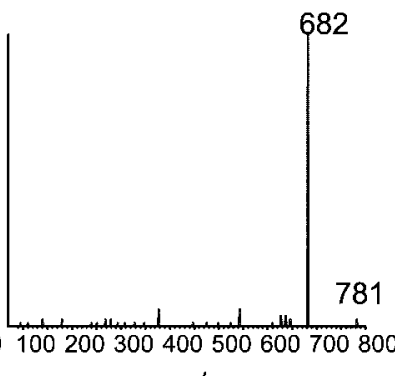

$\mathrm{m} / \mathrm{z}$, amu

81

$\mathrm{m} / \mathrm{z}$, amu

Fig. 1. Positive product ion spectra of clozapine and the test compounds \# 1 through \# 9. 


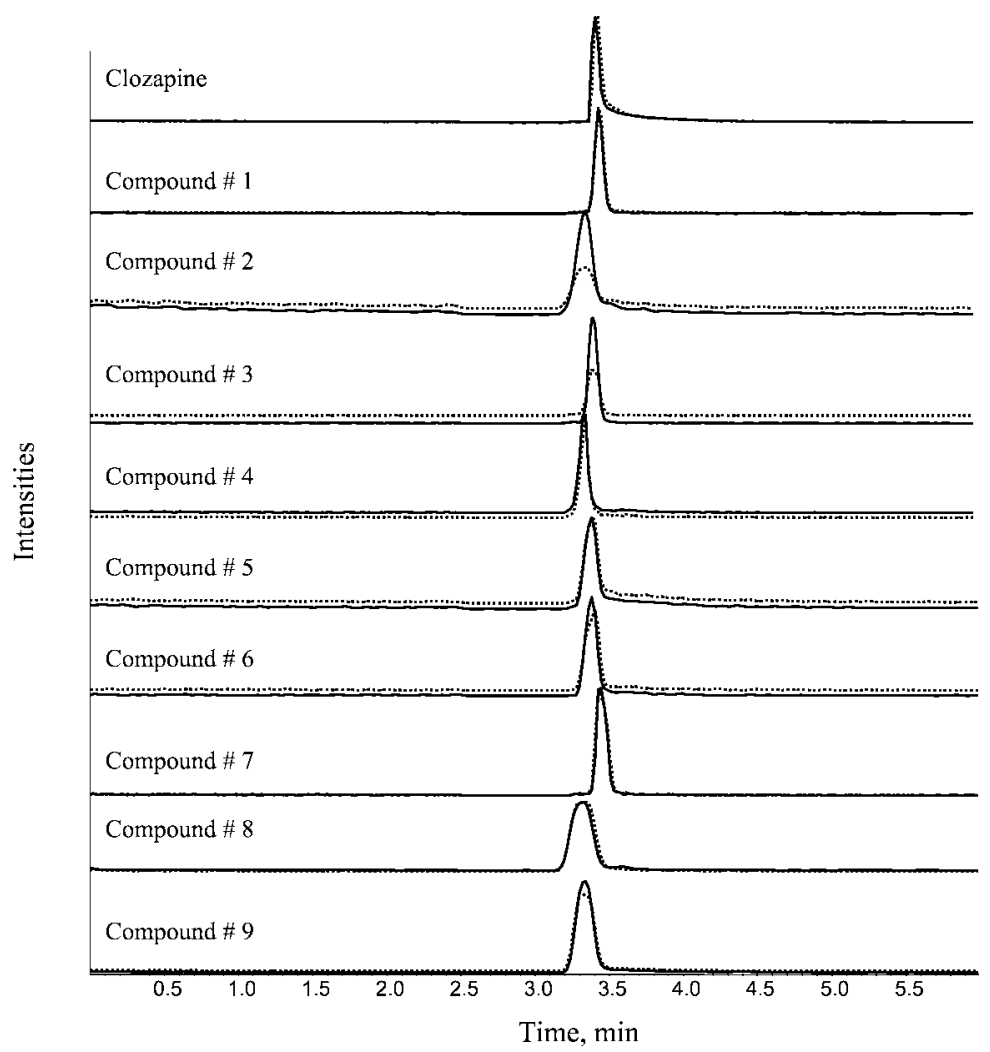

Fig. 2. Reconstructed direct HPLC-MS/MS chromatograms of clozapine and the test compounds \# 1 through \# 9 in the spiked rat plasma after approximately 5-min (solid line) and 180-min (dot line) incubation.

from clogging and to reduce ion source contamination in the mass spectrometer. Direct plasma injection technology coupled with HPLC-MS/MS assay has become an attractive alternative to reduce the time required for tedious sample preparation procedures.

The aim of this study was to extend the utilization of a polymer-coated mixed-function (PCMF) column for direct simultaneous HPLC-MS/MS analysis of stability of multiple compounds in various plasma samples. This proposed procedure eliminates laborious sample collection steps following by time-consuming sample preparation procedures and thereby enhances the efficiency of drug plasma stability studies. The role of the PCMF column is to exclude macromolecules such as protein and to separate small drug molecules by interaction with the small bonded hydrophobic group [8-11].

For the proposed semi-automated procedure, individual rat, mouse, monkey and human plasma samples were spiked with ten test compounds in the thermostatic autosampler (also used as the incubator) which was programmed for sequential injections into the direct HPLC-APCI-MS/MS system. The peak responses of all analytes from the rat, mouse, monkey or human plasma were simultaneously monitored every $7 \mathrm{~min}$. The reconstructed mass chromatograms of all ten compounds of interest after approximately 30-min (solid line) and 180-min (dot line) incubation times are shown in Fig. 2. The retention times and peak shape for all analytes were found to be reproducible throughout the experiment. Also, no interference peak in the mass chromatograms of all analytes from all blank plasma was observed (data not shown). The spiked rat, mouse, monkey and human plasma samples containing 10 analytes were assayed again for 6 repetitive injections after first injection period following the same injection order. The stabil- 


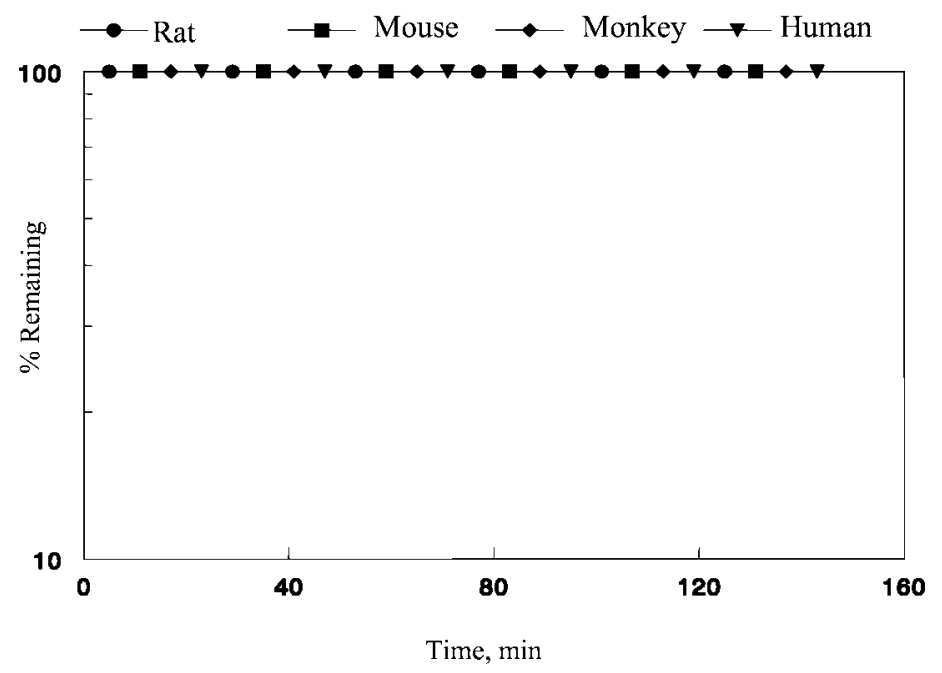

Fig. 3. The stability of clozapine and the test compounds \# 1, \# 4 through \# 9 in the rat, mouse, monkey and human plasma at room temperature $\left(21^{\circ} \mathrm{C}\right)$.

ity of ten test compounds in the rat, mouse, monkey and human plasma as indicated by the changes of peak responses was simultaneously measured. The stability of clozapine, compounds \# 1 and \# 4 through \# 9 in four different plasma samples is plotted in Fig. 3. As shown in Fig. 3, these compounds were found to be stable at room temperature as indicated by the lack of a substantial change in the peak responses from each plasma sample using the proposed approach. Compounds \# 2 and 3 were observed to be stable in mouse, monkey and human plasma within the 3-hour incubation time at room temperature (data not shown) but unstable in the rat plasma, as illustrated in Fig. 2. In Fig. 2, the peak areas of compounds \# 2 and \# 3 in rat plasma decreased as the incubation time increased. The stability results of compounds \# 2 and \# 3 in the rat plasma obtained by the proposed procedure were found to be comparable with those obtained by the traditional sample incubation and collection procedures following by the protein precipitation technique as shown in Fig. 4. In summary, the proposed procedure simultaneously provides the stability results of ten test compounds in four different plasma samples without compromising data quality.

Comparison of the peak area responses of the test compounds in the spiked plasma with those from the spiked supernatant solution with protein precipitation technique (through the PMCF) provided an indication of recovery for each drug candidate for this on-line column extraction procedure as described previously [9-11]. The apparent on-column recoveries of all test compounds (5 sample injections) were studied with the rat, mouse, monkey and human plasma samples spiked at the $250 \mathrm{ng} / \mathrm{ml}$ concentration level and found to be greater than $90 \%$ with \%CV less than 7 .

The accuracy and reproducibility of the analytical results obtained by HPLC-MS/MS method is often affected by the degree of ionization suppression from sample matrix that vary with different biological fluids, sample preparation methods and ionization techniques $[13,14]$. In order to observe the matrix ionization suppression effect for the proposed direct HPLC-MS/MS analyses of plasma samples, we monitored the APCI responses for all ten compounds using the post-column infusion scheme [13,14]. The differences between the extracted infusion mass chromatograms from a mobile phase injection and plasma injection are considered to be caused by the matrix effect resulting from interference materials in the plasma samples eluting from the PMCF columns at various times. Any change in consistent APCI 


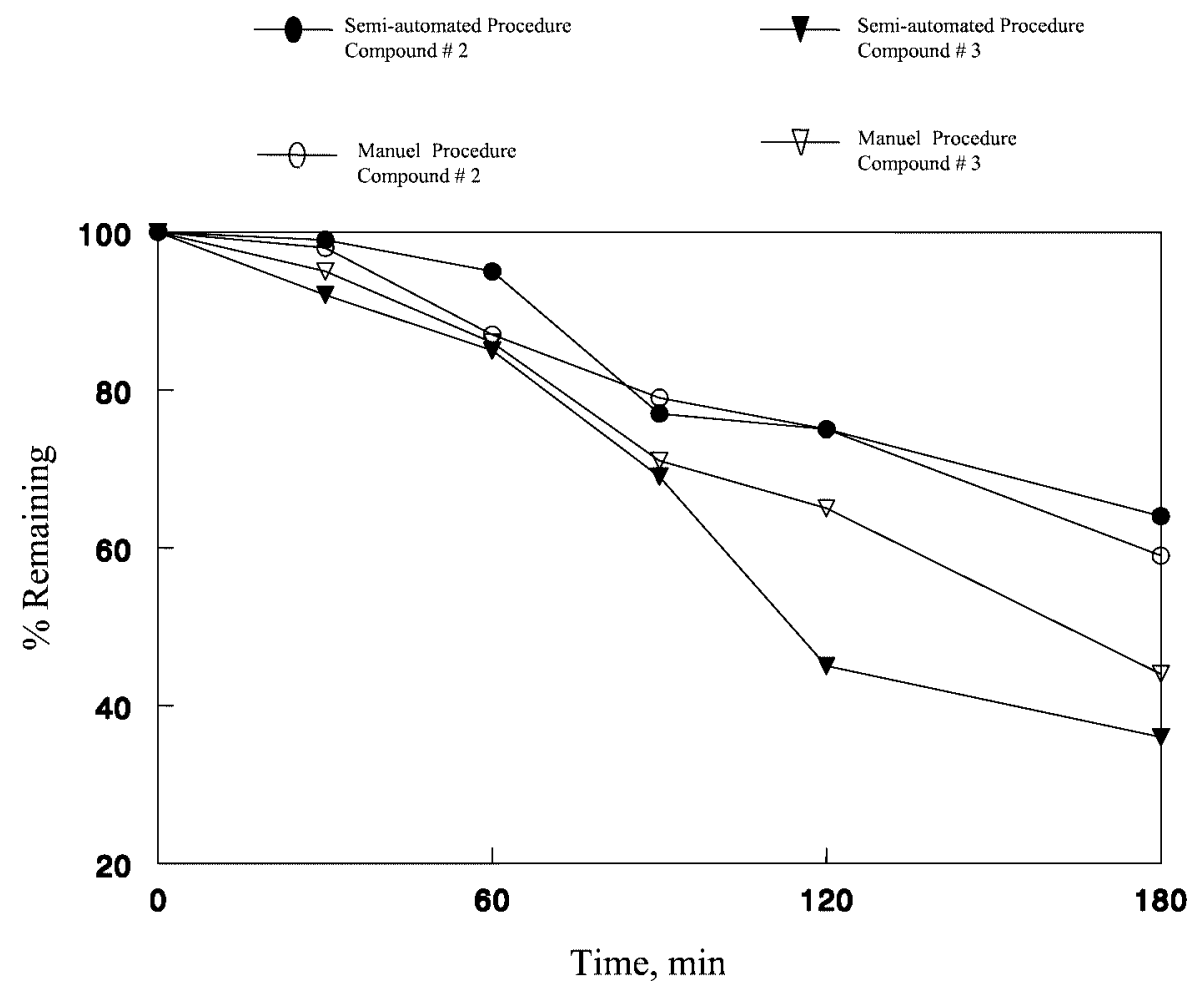

Fig. 4. Comparison of stability results of the test compounds \# 2 and \# 3 in rat plasma obtained by the proposed cassette assay and by the traditional single component incubation procedure.

responses of the infused compounds monitored after the divert valve was switched to the mass spectrometer were presumed to be due to ionization suppression caused by endogenous molecules from the plasma samples which eluted from the HPLC columns. The mixture of the ten analytes was continuously infused into mass spectrometer combined with HPLC effluent when mobile phase B started. The infusion mass chromatograms of all analytes after either $10 \mu \mathrm{l}$ of mobile phase or rat plasma injection into PMCF column are given in Fig. 5. This test was also applied to mouse, monkey and human plasma samples (data not shown). The objective of the post-column infusion experiments was to access the extent of the matrix effect time window. For accurate quantitative determination, it is strongly recommended that the retention times of all analytes should be in the chromatographic region of little or no matrix ion suppression. No difference between these infusion mass chromatograms was observable suggesting that the endogenous interference components were simultaneously washed out with the other macromolecules such as protein in the plasma.

\section{Conclusions}

A direct bioanalytical approach based on a cassette assay technique combined with a mixed-function column LC-APCI/MS/MS has been demonstrated for the plasma stability studies of drug candidates. The proposed ten-in-one approach should prove to be reliable as screen-type assay for high throughput drug stability measurement in plasma and showed identical accuracy of analytical results and ten times greater sample throughput as the conventional single assay method. This allows us to simultaneously 


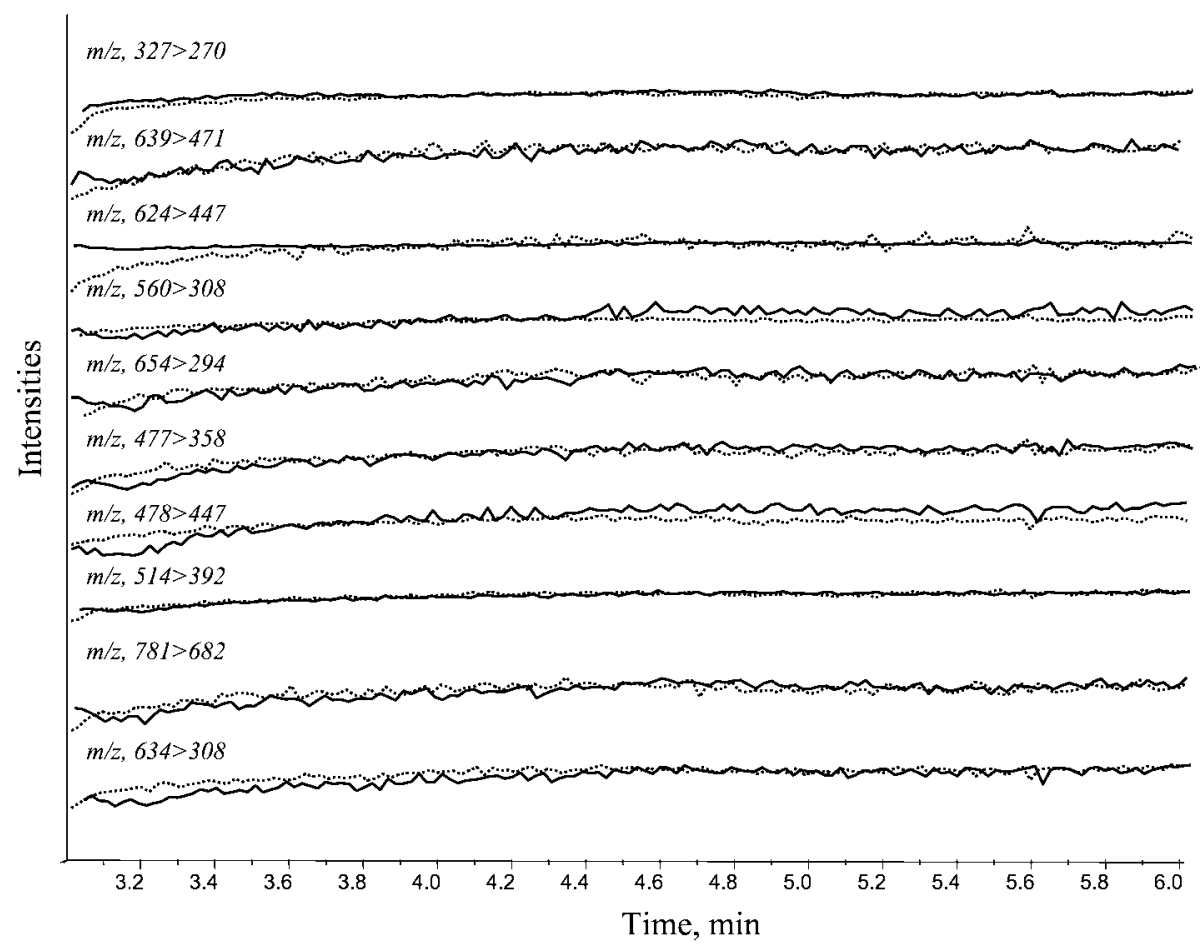

Fig. 5. The reconstructed infusion mass chromatograms of clozapine and the test compounds \# 1 through \# 9 (from top to bottom) after injection of the mobile phase B (dot line) and rat plasma (solid line).

obtain the stability results of ten drug candidates in four different plasma samples in one semi-automated procedure.

\section{Acknowledgements}

The authors thank our Medicinal Chemistry group for synthesizing the drug discovery compounds used in this work.

\section{References}

[1] M.E. Abdel-Hamid, I.O. Edafiogho and H.M. Hamza, J. Pharmaceut. Biomed. Anal. 27 (2002), 225.

[2] M.P. Elizalde-Gonzalez, J. Mattusch and R. Wennrich, J. Chromatography A 828 (1998), 439.

[3] S.X. Peng, M.J. Strojnowski and D.M. Bornes, J. Pharmaceut. Biomed. Anal. 25 (1999), 343.

[4] E. Pop, S. Rachwal, J. Vlasak, A. Zharikova and L. Prokai, J. Pharmaceut. Sci. 88 (1999), 1156.

[5] G. Wang, Y. Hsieh, K.-C. Cheng, J. Lau, K. Ng, W.A. Korfmacher and R.E. White, J. Chromatography B (2002) 451, submitted for publication.

[6] M. Jemal, M. Huang, X. Jiang, Y. Mao and M. Powell, Rapid Commun. Mass Spectrom. 13 (1999), 2125.

[7] J.T. Wu, H. Zeng, M. Qian, B.L. Brogdon and S.E. Unger, Anal. Chem. 72 (2000), 61.

[8] Y. Hsieh, M. Bryant, G. Gruela, J. Brisson and W.A. Korfmacher, Rapid Commun. Mass Spectrom. 14 (2000), 1384.

[9] Y. Hsieh, J. Brisson, K. Ng, R. White and W.A. Korfmacher, The Analyst 126 (2001), 1239.

[10] Y. Hsieh, J. Brisson, K. Ng and W.A. Korfmacher, J. Pharmaceut. Biomed. Anal. 27 (2002), 285.

[11] Y. Hsieh, M. Bryant, J. Brisson, K. Ng and W.A. Korfmacher, J. Chromatography B 767 (2002), 353.

[12] Y. Hsieh, K. Ng and W.A. Korfmacher, Am. Pharmaceut. Rev. (2002), in press. 
[13] Y. Hsieh, M. Chintala, H. Mei, J. Agans, J. Brisson, K. Ng and W.A. Korfmacher, Rapid Commun. Mass Spectrom. 15 (2001), 2481

[14] Y. Hsieh, J. Brisson, G. Wang, K. Ng and W.A. Korfmacher, J. Pharmaceut. Biomed. Anal. (2003) in press.

[15] M. Liu, M.S. Bryant, J. Chen, S. Lee, B. Yaremko, P. Lipari, M. Malkowski, E. Ferrari, L. Nielsen, N. Prioli, J. Dell, D. Sinha, J. Syed, W. Korfmacher, A. Nomeir, C. Lin, L. Wang, A. Taveras, R. Doll, G. Njoroge, A. Mallams, S. Remiszewski, J. Catino, V. Girijavallabhan, P. Kirschmeier and R. Bishop, Cancer Res. 58 (1998), 4947.

[16] R.E. White and P. Manitpistkul, Drug Metabolism and Disposition 29 (2001), 957.

[17] L.W. Frick, K.K. Adkison, K.J. Wells-Knecht, P. Woollard and D.M. Higton, Pharmaceut. Sci. Technol. Today, 1 (1998), 12.

[18] F. Beaudry, J.C. Yves Le Blanc, M. Coutu and N.K. Brown, Rapid Commun. Mass Spectrom. 12 (1998), 1216.

[19] H. Bu, M. Poglod, R.G. Micetich and J.K. Khan, Rapid Commun. Mass Spectrom. 14 (2000), 523.

[20] W.A. Korfmacher, K.A. Cox, M. Bryant, J. Veals, K. Ng, R. Watkins and C. Lin, Drug Discovery Today 2 (1997), 532. 


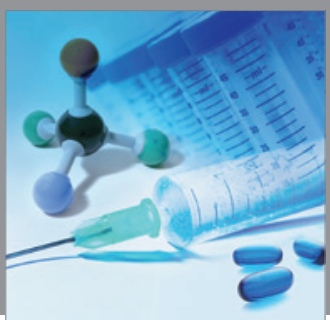

International Journal of

Medicinal Chemistry

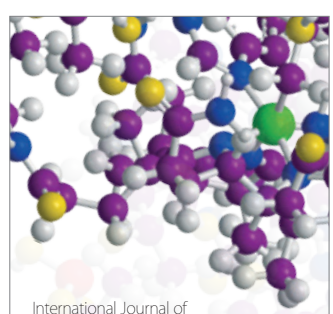

Carbohydrate Chemistry

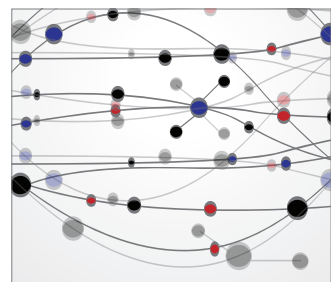

The Scientific World Journal
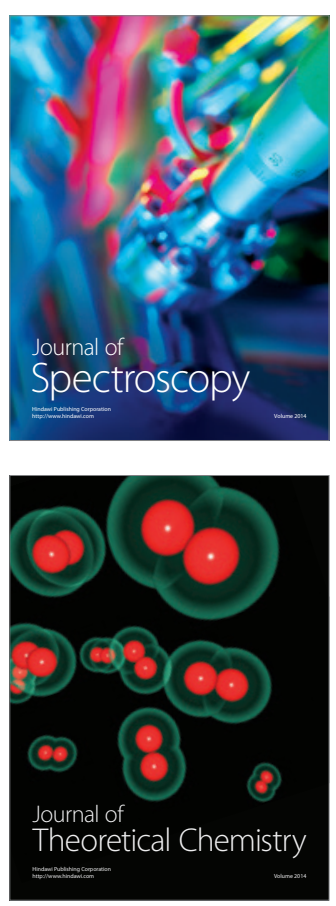
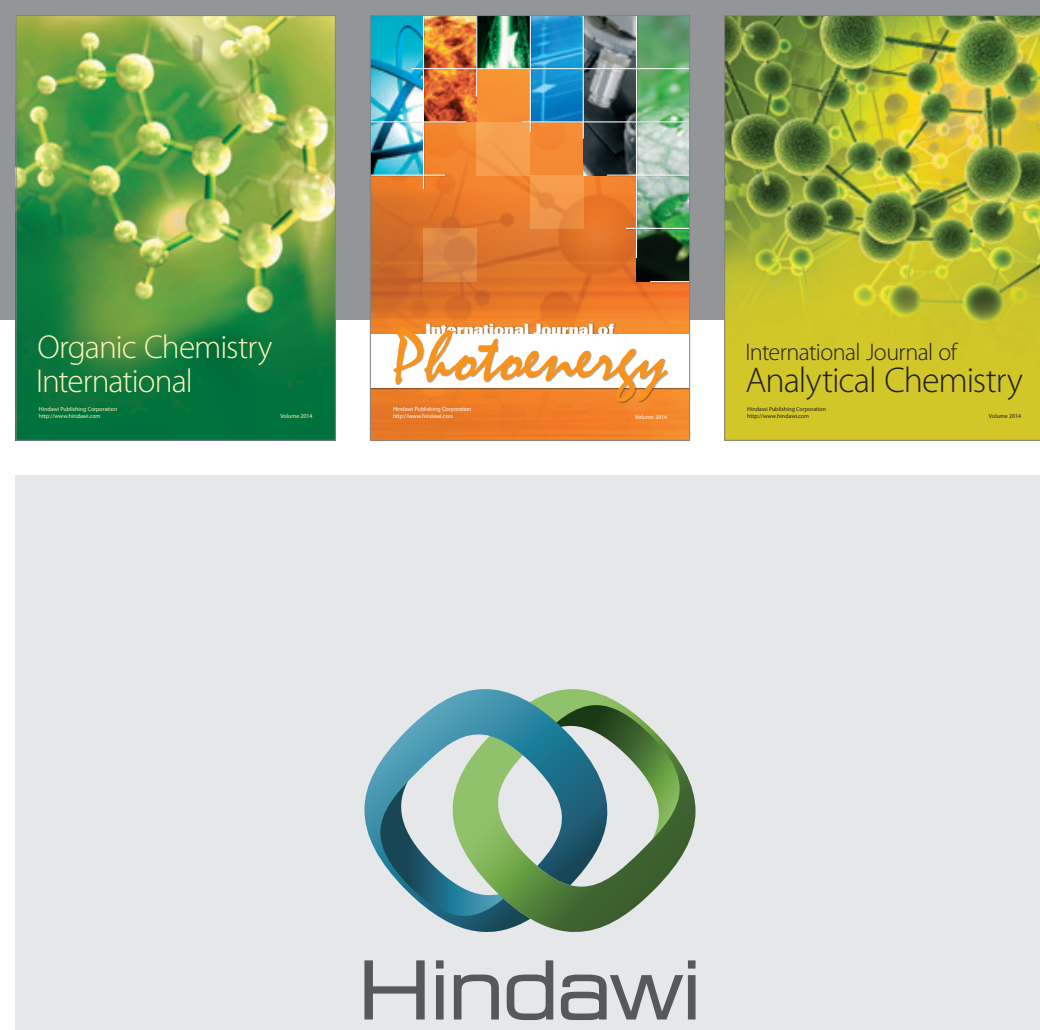

Submit your manuscripts at

http://www.hindawi.com
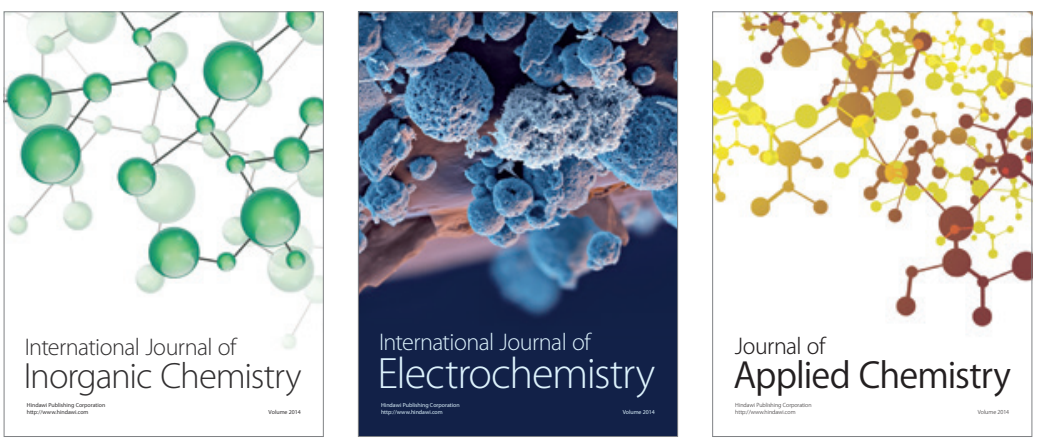

Journal of

Applied Chemistry
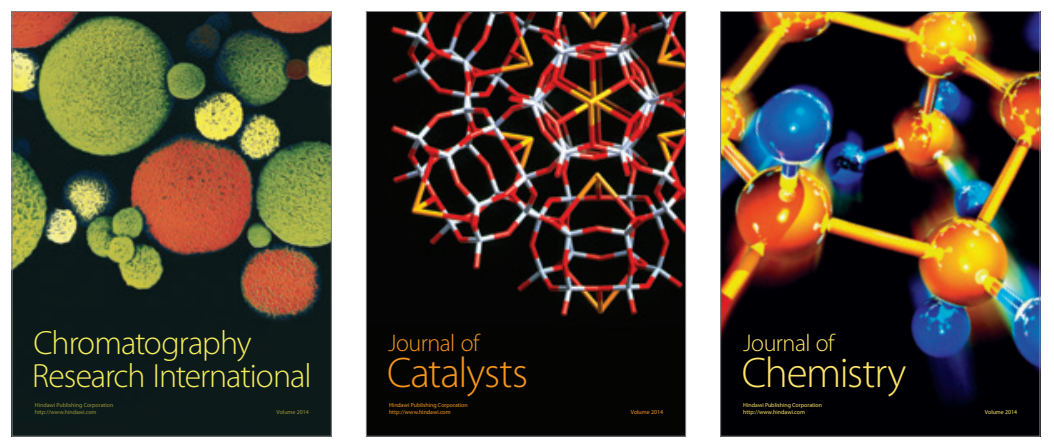
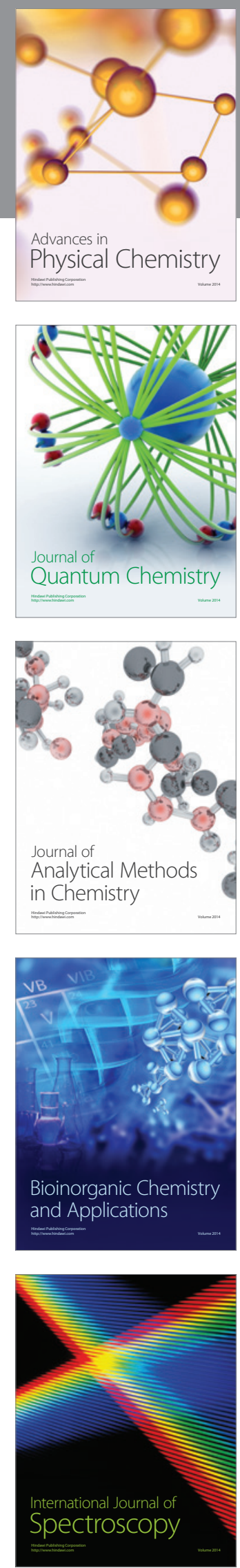